\title{
Illusory Percepts from Auditory Adaptation
}

\author{
Lucas C. Parra* \\ Biomedical Engineering Department, City College of New York, NY 10031, USA \\ Barak A. Pearlmutter ${ }^{\dagger}$ \\ Hamilton Institute, National University of Ireland Maynooth, Co. Kildare, Ireland
}

(Accepted for publication in JASA November 20, 2006)

\begin{abstract}
Phenomena resembling tinnitus and Zwicker phantom tone are seen to result from an auditory gain adaptation mechanism that attempts to make full use of a fixed-capacity channel. In the case of tinnitus, the gain adaptation enhances internal noise of a frequency band otherwise silent due to damage. This generates a percept of a phantom sound as a consequence of hearing loss. In the case of Zwicker tone, a frequency band is temporarily silent during the presentation of a notched broad-band sound, resulting in a percept of a tone at the notched frequency. The model suggests a link between tinnitus and the Zwicker tone percept, in that it predicts different results for normal and tinnitus subjects due to a loss of instantaneous nonlinear compression. Listening experiments on 44 subjects show that tinnitus subjects (11 of 44) are significantly more likely to hear the Zwicker tone. This psychoacoustic experiment establishes the first empirical link between the Zwicker tone percept and tinnitus. Together with the modeling results, this supports the hypothesis that the phantom percept is a consequence of a central adaptation mechanism confronted with a degraded sensory apparatus.
\end{abstract}

PACS numbers: 4366.Ki,4366.Mk,4366Ba,4366Dc

\section{INTRODUCTION}

\section{A. Illusory auditory percepts}

Tinnitus is the perception of a phantom sound often associated with hearing $\operatorname{loss}^{1}$. Mild tinnitus is rather common, reported by many subjects after a few minutes in a quiet environment ${ }^{2,3}$. The subjective sound varies, often described as a "buzz", "ring", "hiss", "hum", or the like. Severe tinnitus is almost always indicative of hearing loss ${ }^{4}$, with the pitch of the phantom sound generally corresponding to the frequencies of hearing loss ${ }^{5}$ and occurring predominantly at sharp edges of high-frequency $\operatorname{loss}^{1}$. To date, a variety of therapeutic approaches to alleviate tinnitus have given mixed results ${ }^{3,6}$. It is therefore generally assumed that tinnitus may be the result of multiple physiological causes ${ }^{7}$. It is believed that in most cases the tinnitus percept does not originate immediately at the cochlea. Instead it has often been associated with adaptive phenomena in the central nervous system ${ }^{4,8}$. A variety of models for the physiological origin of this form of central tinnitus have been proposed ${ }^{9,10}$.

The Zwicker tone, an auditory perceptual illusion named after the scientist who first characterized it, is a transient phantom sound that is perceived by most subjects after a notched broad-band signal ${ }^{11-13}$. The frequency of the illusory sound falls within the notched fre-

\footnotetext{
*Electronic address: parra@ccny. cuny.edu

$\dagger$ Electronic address: barak@cs.nuim.ie; URL: http://www.bcl. hamilton.ie/ barak/
}

quency band, and is closer to the lower stop-band edge for a wide-band notch. The strength and duration of the Zwicker tone percept depends on stimulus conditions and is quite variable across subjects ${ }^{14,15}$. In fact, in the study presented below, some subjects with normal hearing did not hear a Zwicker tone.

Despite their apparent similarity, the exact relationship between the Zwicker tone and tinnitus is not well established. This paper (a) presents a conceptual model, fleshed out mathematically, that gives a common explanation for both phenomena; (b) shows that this model predicts a linkage between tinnitus and Zwicker tone; and (c) exhibits psychophysical data which matches the prediction of the model. The psychophysical data does support the proposed model, but also considered in isolation constitutes a novel empirical link between tinnitus and the Zwicker tone.

The remainder of the paper starts, in Section I.B, with a review of the standard theoretical understanding of simple sorts of adaptation as mechanisms for matching sensory statistics. Section I.C motivates a model of auditory adaptation based on these principles, and Section II instantiates the model mathematically. Simulations of this model in Section III suggest an empirical link between tinnitus and the Zwicker tone. This link is confirmed by the psychosocial observations reported in Section IV.

Taken together, the experimental and modeling results support the hypothesis that tinnitus is a consequence of a gain adaptation mechanism that is confronted with hearing loss and an associated loss of nonlinear compression. Section V presents additional experimentally testable predictions that follow directly from this the- 
ory. Specifically, this predict a relationship between the strength of nonlinear auditory phenomena such as combination tones ${ }^{16-18}$ with the sensitization observed following notched noise ${ }^{19,20}$. Finally, it is noted that elevated hearing thresholds and loss of non-linear compression are easily measured and may be partially restored with compressive hearing aids. This suggests a potential diagnosis and treatment option for those cases of tinnitus that can be linked to this particular form of hearing deficit.

\section{B. Adaptation and Optimality}

Gain and contrast adaptation is a common strategy of the perceptual system to match a large dynamic range of natural signals to the limited dynamic range of sensors and neurons ${ }^{21,22}$. Perhaps the best known gain adaptation mechanism is the closing of the iris when stepping from a dark environment into a bright one. The analogous effect in hearing is the acoustic middle-ear reflex, which mechanically attenuates sound transmission to the cochlea in response to loud sounds ${ }^{8}$.

Adaptation to changes in stimulus statistics is a ubiquitous and long-studied phenomenon in the nervous system $^{23}$. Visual neurons in the retina and visual cortex adjust the gain of their transfer functions to maintain a high sensitivity at varying luminance contrast levels ${ }^{24-26}$. This allows the visual system to operate well under drastically varying external conditions. In the auditory system, adaptation is observed at various levels. Efferent feedback to outer hair cells are thought to control the gain of cochlear amplification ${ }^{27}$, while auditory nerve fibers are known to adapt their firing rate at various time scales $^{28,29}$. Finally, inferior colliculus neurons have been shown to adjust their response thresholds and gains to optimally encode variations in the auditory stimulus ${ }^{30,31}$.

The main theoretical contribution of this paper is to demonstrate that some illusory auditory percepts can be explained as direct consequences of gain adaptation and internal noise in the presence of hearing loss. Gain adaptation and noise are basic features of the auditory processing stream ${ }^{8,32,33}$. Since gain adaptation may operate at various levels of processing, a simple and generic model is constructed. It is then shown that, after gain adaptation, model frequency bands with reduced external input (due to permanent hearing loss or temporary deprivation) show enhanced steady-state activity resembling phantom sounds.

A generic argument is purposefully made, in order to be applicable at many stages of auditory processing, rather than suggesting which area or areas actually subserve this functionality. The auditory nerve is, however, discussed as one potential site where such a mechanism may play a role. The model is sufficiently generic that is to be expected that similar phenomena would be exhibited by any system in its broad general class: systems that perform local gain adaptation in the context of a global estimate of the stimulus energy.

\section{Information bottleneck and gain adaptation}

The cochlea transforms acoustic signals into neuronal activity by decomposing the signal into its various frequency components, which are then transmitted by the auditory nerve to the midbrain. The signal intensity in different frequency bands is encoded in the firing of different neurons which project into the auditory nerve. However, the dynamic range of the external stimuli is known to be much larger than the dynamic range of this neuronal activity.

Transmitting auditory information through this information bottleneck therefore requires adaptive mechanisms. The nervous system has developed various strategies to cope with this problem including, in particular, gain adaptation. It can be argued that the main goal of the adaptive mechanism should be to transform the signal in different frequency bands into independent channels with optimally matched dynamic ranges. In this view, gain adaptation accomplishes two tasks. First, it adjusts signal variance to the effective dynamic range of each frequency channel, thus optimizing the information capacity in each frequency channel ${ }^{22}$. Second, it removes redundancy across channels. Most acoustic signal have significant redundancy across frequency bands due to frequency co-modulation - the simultaneous increase and decrease of amplitude in multiple bands ${ }^{34}$. In fact, humans can understand spoken language with as few as four distinct frequency bands, supporting the notion that information is redundantly encoded across different frequencies ${ }^{35}$. By normalizing signal power, channels become more independent ${ }^{36}$. A similar mechanism for reducing redundancy by divisive normalization has been proposed for visual processing ${ }^{37-39}$ and can be used for image compression ${ }^{40}$.

A channel with fixed dynamic range will communicate maximum information if the transfer function matches the cumulative density function (CDF) of the input variable $^{41}$. In particular, the threshold and slope of the transfer function should match the mean and variance of the data. By adjusting the mean and/or variance of the input, one can optimize the information transmission for a given transfer function. The first-order correction is achieved by adjusting the variance (or power) of the signal to match the transfer function. ${ }^{42}$

\section{METHODS (MODELING)}

\section{A. Gain adaptation model}

The model assumes (a) that instantaneous signal power is transduced by the cochlea into a neuronal signal, and (b) that neuronal transmission has some inherent noise such as spontaneous background firing. $|S(\omega, t)|^{2}$ denotes the instantaneous power of the stimulus in frequency band $\omega$ at time $t$. If the random neuronal noise $N(\omega)$ is independent of the stimulus, the neuronal repre- 
sentation of signal power $P(\omega, t)$ in each frequency band is simply the sum of the signal and noise,

$$
P(\omega, t)=|S(\omega, t)|^{2}+N(\omega)
$$

It seems natural to suggest that gain adaptation should normalize this signal so that it lies within a limited dynamic range. The normalization should occur separately for each frequency band, using a long-term average of stimulus power. Computationally, the simplest estimate of signal power is a running average, which updates the long-term power estimate with the currently observed instantaneous power values. In a discrete-time formulation this is given by

$$
\bar{P}(\omega, t)=(1-\mu) \bar{P}(\omega, t-\Delta t)+\mu P(\omega, t)
$$

where $P(\omega, t)$ is the neuronal representation of instantaneous stimulus power, $\bar{P}(\omega, t)$ is an estimate of the long-term average power, and $\mu<1$ is a fraction that captures how much the current estimate contributes to the long-term average. ${ }^{43}$ This is equivalent to a temporal integration of instantaneous powers with an integration window $w_{n}$ :

$$
\bar{P}(\omega, t)=\sum_{n=0}^{\infty} w_{n} P(\omega, t-n \Delta t)
$$

where the weights decay exponentially, $w_{n} \propto(1-\mu)^{n}$. In order to ensure that the integration window is normalized, i.e., that $\sum_{n} w_{n}=1$, a constant is introduced so $w_{n}=\mu(1-\mu)^{n}$. The integration time can be characterized by a time constant $\tau=\Delta t / \mu$.

Now let the normalization gain for each band be

$$
G(\omega, t)=\frac{1}{\bar{P}(\omega, t)}
$$

so the normalized neuronal representation of stimulus power is

$$
E(\omega, t)=G(\omega, t) P(\omega, t)
$$

This normalization ensures that the neuronal signal is within some fixed dynamic range, and also removes the cross-frequency redundancy of co-modulated power envelopes.

As seen below, these simple assumptions motivated by considerations of optimal signal processing give rise to illusory percepts resembling tinnitus and Zwicker tone in response to a reduced input in a given frequency band.

\section{B. Sensitivity and hearing loss}

The perceived signal intensity in each frequency band is affected by the sensitivity of the cochlea at that band. This is expressed by some gain function $H(\omega)$, with $H(\omega)|S(\omega, t)|^{2}$ replacing $|S(\omega, t)|^{2}$. Hearing loss is modeled by reducing $H(\omega)$ for the damaged bands. The broadening of the bandwidth associated with hearing loss has not been modeled here.

\section{Compression of the cochlear amplifier}

Note that this simple model is linear in power, and therefore does not include the nonlinear compression typically found for an intact cochlea ${ }^{44}$. Outer hair cells are thought to actively amplify faint sounds with high gain, whereas loud sounds are transduced with a lower gain ${ }^{45}$. This non-linear amplification leads to a compression of dynamic range. Theoretical models ${ }^{46}$ as well as psychophysical experiments ${ }^{47}$ describe the compressive effect as power laws with powers between 0.3 and 0.5 for normal hearing. A power law is easily included in the present model with a power factor $\beta$. Together with the sensitivity discussed above, this gives

$$
P(\omega, t)=H(\omega)|S(\omega, t)|^{2 \beta}+N(\omega)
$$

A power factor $\beta<1.0$ reflects normal compression of the cochlear amplifier, whereas $\beta=1.0$ reflects a lack of active amplification ${ }^{48}$ resulting in a sharper increase in firing rate with increasing signal power observed for the damaged cochlea ${ }^{49}$.

\section{Lateral inhibition}

The pitch of tinnitus is sometimes perceived at pronounced edges of hearing loss ${ }^{10,50}$. Similarly, for wideband notched noise the Zwicker tone is perceived preferentially at the lower edge of the notched band ${ }^{13}$. To explain this phenomenon most models of tinnitus and Zwicker tone include some form of lateral inhibition ${ }^{10,11,51,52}$. In fact, they require asymmetric inhibition whereby high frequencies tones inhibit lower frequency units. Such asymmetric lateral inhibition has been reported in the auditory nerve, cochlear nucleus ${ }^{53}$, inferior colliculus ${ }^{54}$, and auditory cortex ${ }^{55}$. To demonstrate the effect of asymmetric lateral inhibition in the present model, the excitation can be modified

$$
\log |S(\omega, t)|=\sum_{\Delta \omega} v(\Delta \omega) \log |S(\omega-\Delta \omega, t)|
$$

with $v(\Delta \omega)$ representing some interactions of frequency bands separated by $\Delta \omega$. A simulation incorporating such asymmetric lateral inhibition is shown in Figure 2, which corresponds to lateral inhibition prior to adding noise and gain adaptation (see discussion in Section V.C). Similar effects are obtained if the asymmetric lateral inhibition is introduced after gain adaptation.

\section{E. Recovered signal}

To interpret the neuronal representations after gain adaptation, the neuronal model signal can be used to construct an estimate of the original signal. This step may seem artificial, as the nervous system does not need to regenerate the original signal in order to perceive it. 

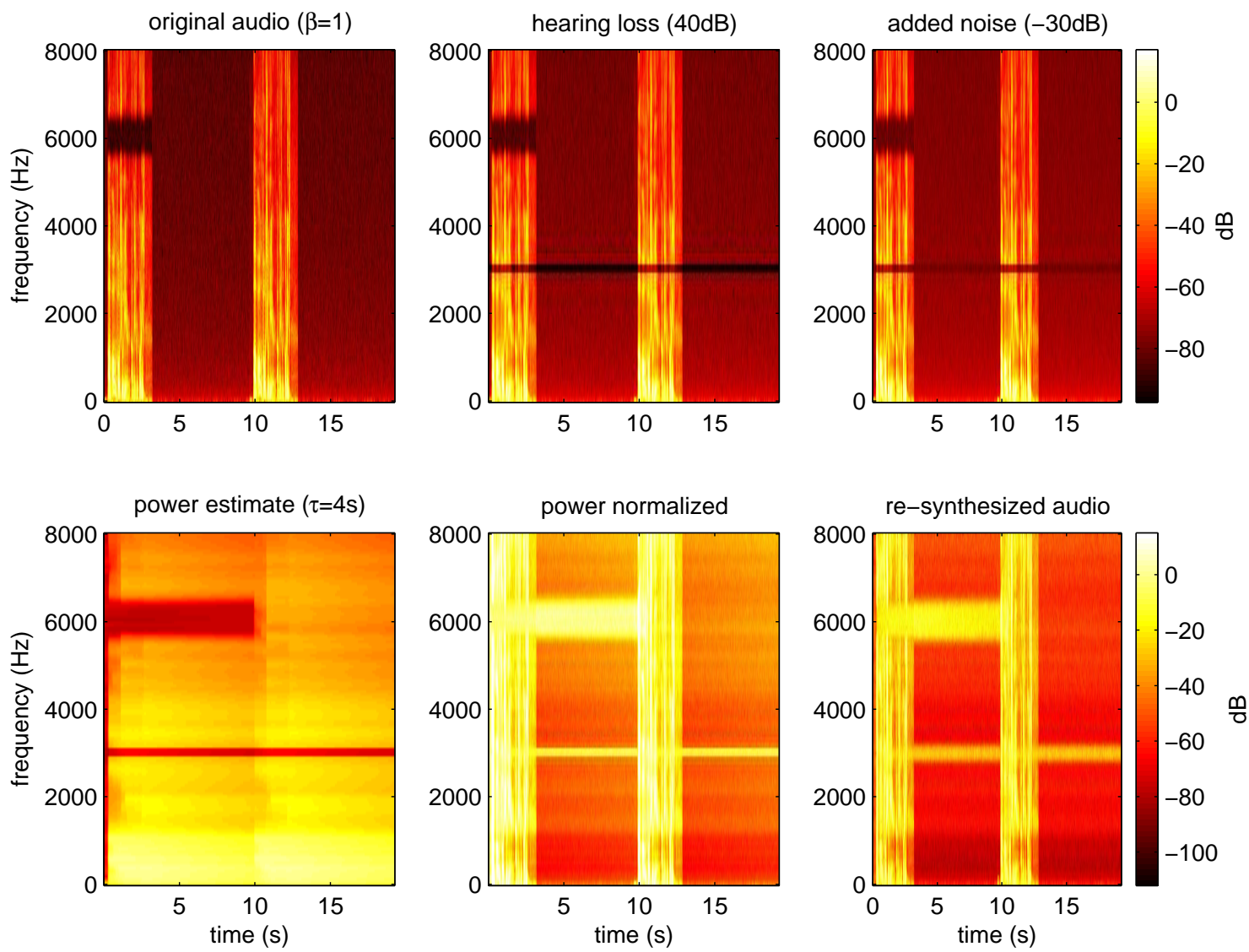

FIG. 1. Stages of processing showing how phantom auditory percepts are generated by gain adaptation. A constant tone resembling tinnitus is generated at the frequency of hearing loss $(3 \mathrm{kHz}$ here $)$. A transient tone resembling the Zwicker tone is generated following the notched sound $(6 \mathrm{kHz}$ here). The auditory signal is first decomposed into a time-frequency representation (top left). Frames of $16 \mathrm{~ms}$ (256 samples at a $16 \mathrm{kHz}$ sampling rate) around time $t$ are windowed with a Hanning window and Fourier transformed to obtain 128 frequency amplitudes $|S(\omega, t)|$ (top left) and phases $\arg (S(\omega, t)$ ) (not shown). Image intensity represents power in $\mathrm{dB}$ using the same color map for each row. Perceived powers (top center) are given by $H(\omega)|S(\omega, t)|^{2}$. Noise with a power profile $N(\omega) \propto 1 / \omega$ is added to the perceived powers, giving the signal $P(\omega, t)$ (top right) according to Equation (6). The gain and equalized signal powers (bottom left and center) are computed with Equations (4) and (5) using a time constant of $\tau=4000 \mathrm{~ms}$. The original signal powers are estimated from this activity using Equation (8) and the signal is re-synthesized. A spectrogram of this re-synthesized signal is shown on the bottom right. (The re-synthesized sounds are available at http://newton.bme.columbia.edu/ lparra/tinnitus/.)

Rather, this neuronal representation either itself constitutes, or is the precursor of, perception. Regardless of the physical basis of auditory qualia, it can be argued that if the representation is altered such that the stimulus can not be regenerated, even approximately, then the percept must be equivalently distorted, and that the reconstruction technique provides an intuitive way to measure and visualize the distortion of the neuronal representation. Here, this method is used to show that the regenerated signals after gain adaptation exhibit artifacts that would be perceived as phantom sounds.

To interpret (and hear) the adjusted signal, an attempt must be made to reproduce the original signal $S(\omega, t)$ from $E(\omega, t)$. If the activity in band $\omega$ is associated with $E(\omega, t)$ and the total power of the signal,
$P(t)=\sum_{\omega} P(\omega, t)$, can be used resulting in

$$
|\hat{S}(\omega, t)|^{2}=P(t) E(\omega, t)
$$

The assumption here is that the system does not know the varying gain it has applied to the signal. However, it does know the overall loudness of the signal as represented by $P(t)$.

Note that gain normalization as proposed here removes the common power of the signal on the time scale $\tau$, so the overall loudness of a signal is therefore no longer reflected in the individual perceptual channels. Stationary silence would therefore be indistinguishable from loud stationary noise. The postulated mechanism therefore implies that the common signal power $P(t)$ must be separately encoded. For a frequency co-modulated signal, power 
is redundantly distributed across bands. Removing this co-modulation removes the redundancy and makes more efficient use of the information capacity of the channel. Communicating overall power as a variable separate from the power fluctuation in each frequency band is therefore a more efficient use of channel information capacity.

Finally, in order to listen to the recovered signal, a time-domain signal must be generated from the timefrequency powers. The powers give amplitude but not phase information. This is a common problem in speech and sound synthesis. A standard engineering solution to this problem is to reuse the phase that was obtained when analyzing the original signal. This is done using a conventional overlap-add procedure: powers are combined with the original phase, $\arg (S(\omega, t))$, inverse Fourier transformed, multiplied with a Hanning window, and added in half-overlapping frames. If the powers have not changed significantly, the resulting signal is perceptually similar to the original.

\section{MODELING RESULTS}

Hearing damage can be simulated by reducing the sensitivity $H(\omega)$ in a narrow frequency band. Figure 1 shows the result for a $40 \mathrm{~dB}$ hearing loss at $3 \mathrm{kHz}$ and $-30 \mathrm{~dB}$ internal noise. The lower right panel shows that gain adaptation generates steady-state power at the damaged frequency band. The reconstructed signal ${ }^{56}$ reproduces a sound similar to tinnitus.

The figure also shows the results obtained for a broadband sound with a notched response (power reduced by $40 \mathrm{~dB}$ at $6 \mathrm{kHz}$ ). Power normalization fills in the gap and generates an artificial tone following the notched noise. This is consistent with the Zwicker tone phenomenon. The increased gain following the noise is consistent with the sensitization ${ }^{20}$ and release of masking ${ }^{19}$ observed after notched noise stimuli in psychophysical studies.

The effect of asymmetric lateral inhibition, and of the slope of the band edge, can now be analyzed. Lateral inhibition is used as shown in Figure 2, and given by $v(\Delta \omega)=\delta(\Delta \omega)-c \exp (-\Delta \omega / B) \theta(\Delta \omega)$, where $c$ and $B$ determine the strength and bandwidth of the inhibitory effect, and $\theta(\Delta \omega)$ is a step function that implements the asymmetry. Figure 2 shows that for a sharp band-gap the phantom sound is narrow-band and appears at the lower edge of the band-gap. In fact, the phantom sound is generated even in the absence of the high-pass edge. This is all in agreement with psychophysical reports on Zwicker tone and tinnitus ${ }^{13,50,51}$. Furthermore, under the assumption of lateral inhibition, it is found that a sloping edge will not cause a phantom percept, while an increasing slope will cause a stronger phantom percept. Also note that the pitch of the percept appears at the position of strongest loss and not the onset of the lossedge. These observations are consistent with empirical finding on tinnitus and non-tinnitus subjects with varying hearing loss profiles ${ }^{1}$.
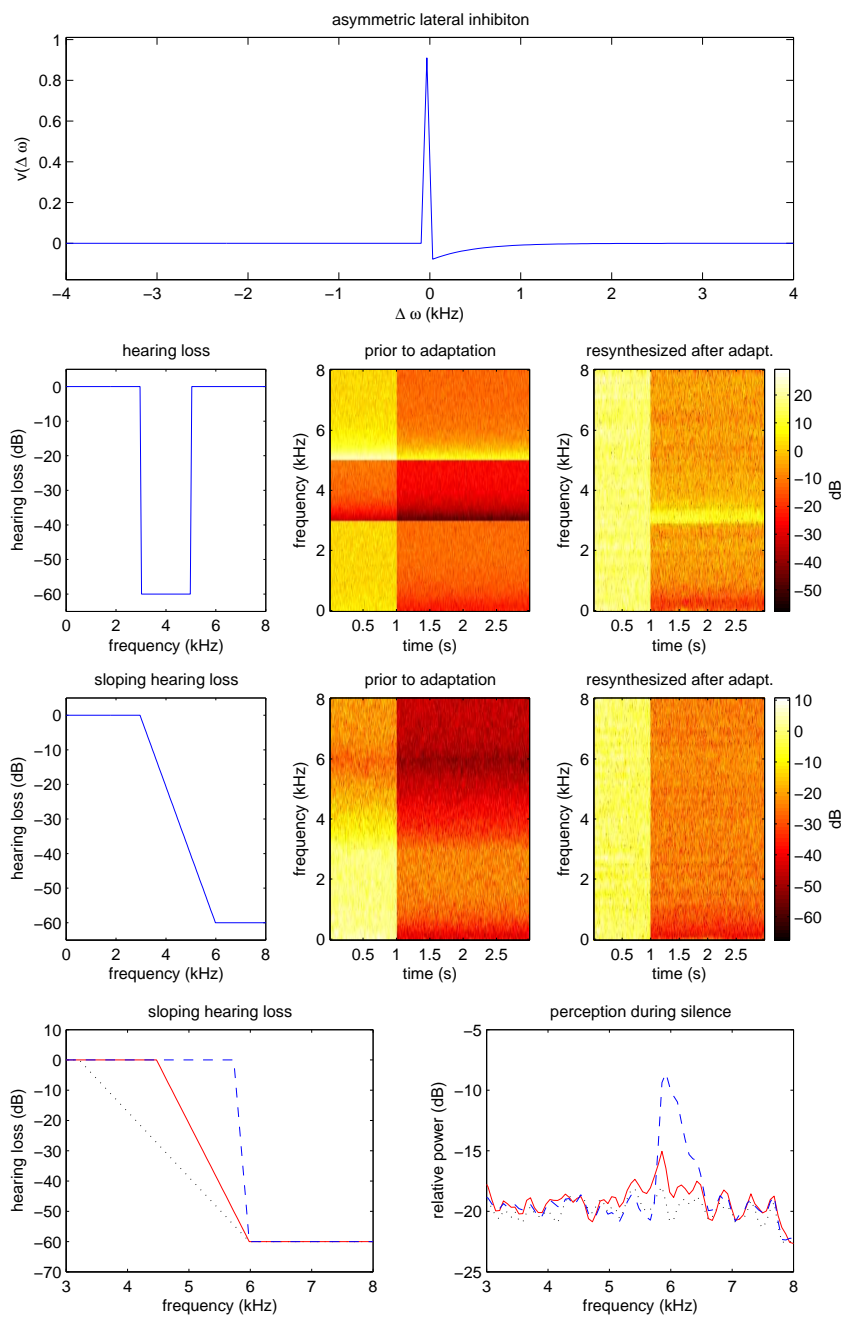

FIG. 2. Effect of asymmetric lateral inhibition and the slope of hearing loss on the tinnitus percept. Top graph: Asymmetric lateral inhibition function $v(\Delta \omega)$. Second row: Loss function $H(\omega)$ with a sharp band-gap (left panel) generates a phantom sound above the lower edge of the gap as evidenced by the spectrogram of the reconstructed signal (right panel). Center panel shows time-frequency representation of the response prior to adaptation. Third row: Panels as in second row, but with a sloping hearing loss. When simulating this with lateral inhibition (as in top graph) sloping loss does not generate a phantom sound (right panel). Fourth row: Comparison of the phantom percept during the silence period (1000-3000 ms) for different slopes of hearing loss. The right panel shows the average power during the $2000 \mathrm{~ms}$ of silence following the $1000 \mathrm{~ms}$ white noise stimulation, and is given in $\mathrm{dB}$ relative to the total power of the preceding noise. An increasingly sharp loss accentuates the phantom percept. The phantom sound is at the lower edge of the loss-band.

The effects of a perceptual frequency scale, where bandwidth increases with center frequency ${ }^{57,58}$, can also be analyzed. The re-synthesized signals show the same qualitative behavior, except that the phantom sound is broadened as a result of the broad bands on the percep- 

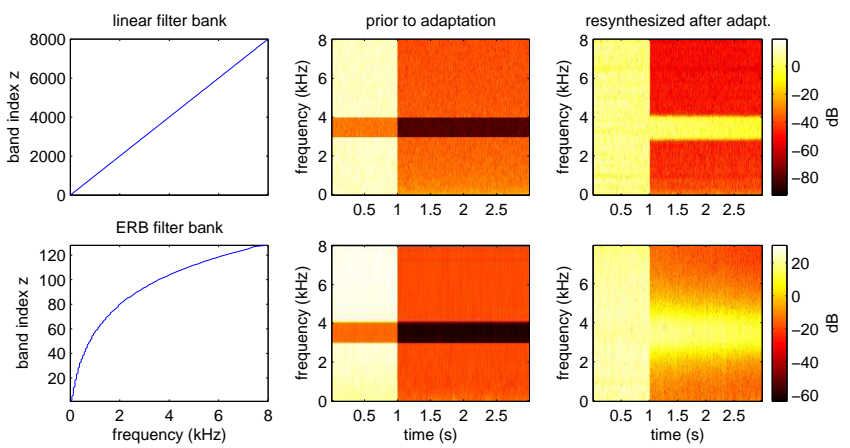

FIG. 3. Effect of perceptual frequency scale. The center column shows a time-frequency representation of the response prior to adaptation, while the right column shows a spectrogram of the reconstructed signal following gain adaptation. Color represents signal intensity in dB. First row: Effect of uniform hearing loss with a linear frequency scale as in Figure 1. Second row: Perceptual frequency bands broaden the effect due to their broad overlapping frequency response. The best frequency of the filter bank is shown on the horizontal axis of the left panel. Compare this to the linear scale on the top-left panel.

tual scale.

The relevant parameters of the present model, most of which are summarized in Equation (6), consist of: the level of signal loss $H(\omega)$, the amount of internal noise $N(\omega)$, the compression factor $\beta$, and the time constant $\tau$ which determines the integration time in Equation (3). Figure 4 shows the effect of each of these parameters on the phantom sounds. The intensity of the phantom sounds increases with the level of internal noise and with the loss of signal intensity, but is fairly independent of $\tau$. It is important to note that the Zwicker tone is decreased in magnitude and duration when considering the effect of non-linear compression of the cochlear amplifier with typical compression factors of $\beta<0.5$.

\section{MODEL PREDICTION AND EVALUATION}

The modeling results shown in Figure 4 (bottom panel) indicate that the Zwicker tone percept is strongest in the case of reduced compression. There is some evidence that tinnitus may be associated with a loss of compression $^{47,48}$, which according to the above model would also accentuate the perception of the Zwicker tone. When normal and tinnitus subjects were tested for differential perception of a Zwicker tone, it was found that in fact the two phenomena were empirically linked.

The perception of the Zwicker phantom tone following a two-second notched noise varied across subjects. Subjects reported different percepts, describing them variously as a "tone", "hiss", or "ringing" lasting a brief moment after the notched noise. More than half the subjects (54\%) perceived a sound of varying strength for different
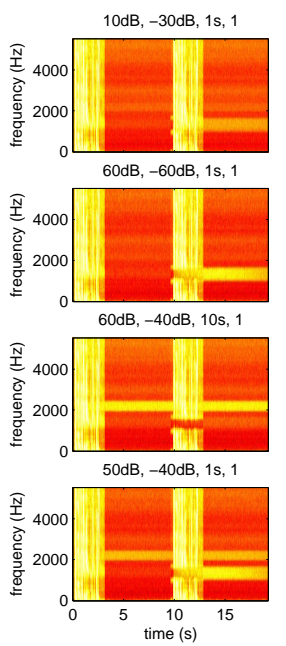

FIG. 4. Dependence of reconstruction on signal loss, noise magnitude, power-averaging time constant $\tau$, and non-linear compression factor $\beta$ (the heading of each panel specifies these parameters in that order). The two phantom percepts occur variably depending on the specific parameters. Importantly, the Zwicker tone is increased with abnormally high $\beta$ corresponding to reduced cochlear compression.

notched-bands, while others did not perceive a phantom tone following any of the notched noise sounds (47\%) (see Methods IV.A).

All subjects were asked if, in their daily lives, they perceived spurious ringing on a regular basis. $25 \%$ of the subjects (11 of 44) responded positively. ${ }^{59}$ There was a correlation between this self-reported tinnitus and the perception of the Zwicker tone $(r=0.42, p=0.004)$. Table $\mathrm{V}$ and figure 5 show that tinnitus subjects are almost certain to hear a Zwicker tone, whereas fewer than half of normal subjects heard a phantom tone following a twosecond notched noise. These numbers show a significant association between self-reported tinnitus and perception of the Zwicker tone ( $p=0.006$, two-tailed Fisher exact test).

\section{A. Methods (Psychophysical)}

Subjects: 44 volunteers were recruited (22 male,

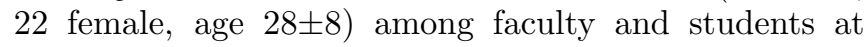
CCNY in accordance with the CCNY IRB guidelines. Subjects gave informed consent prior to experimentation. In this sample there was no significant correlation between the subject's age and self-reported tinnitus, nor between age and the Zwicker percept. The presence of the Zwicker percept was determined with the following procedure.

Zwicker tone perception test: Subjects were tested to determine if they reliably perceived a phantom tone by presenting four different noise sounds in random order. The control sound was white noise, and the other three 


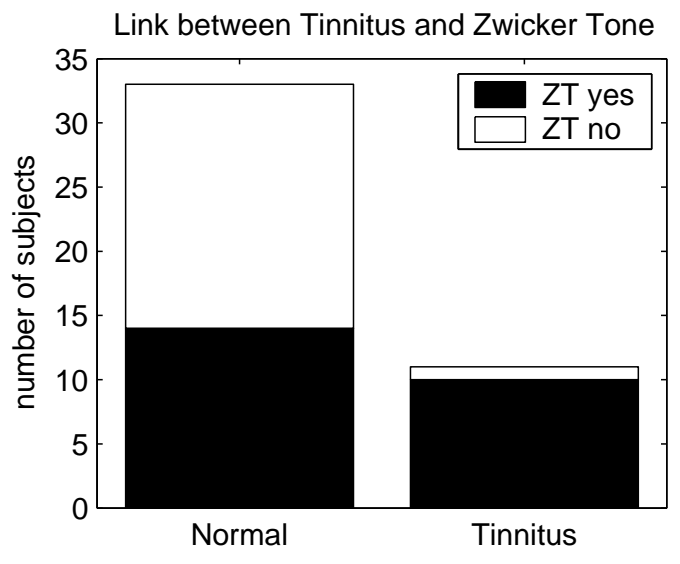

FIG. 5. Number of normal and tinnitus subjects reporting Zwicker tone percept. The Zwicker tone percept was significantly associated with tinnitus self-report $(p<0.01)$.

were notched noise with different notch bands, as described below. Subjects were instructed to report which of the four noises was followed by a perception of some form of ringing, however faint it might be. The percept was considered factual if the subject consistently reported a percept for the same notched sounds (despite the random ordering) but not the white noise. Subjects that did not report any phantom percept, or that gave inconsistent answers to this test, where considered to not perceive the Zwicker tone.

Stimuli: The amplitude of the notched noise rises linearly for $1000 \mathrm{~ms}$, holds for $1000 \mathrm{~ms}$, and decays within $40 \mathrm{~ms}$. The band gap of the notched noise is $4 \mathrm{KHz}$ wide starting at 500, 1000 , or $2000 \mathrm{~Hz}$. The noise sequences where presented with a silence gap of three seconds. The signals were generated on a PC using MATLAB by zeroing the corresponding frequencies in the Fourier domain. They were reproduced using an Audiotrack MAYA44 USB external digital-to-analog converter, and delivered binaurally with audio-technica ATH-M40f headphones at approximately 50-60 dB SPL adjusted for comfort. (Note that standard soundcards in today's PCs can typically not reproduce sharp band-gaps).

\section{DISCUSSION}

Illusory visual percepts were once thought to constitute regimes where the visual system breaks down and fails to process the data appropriately. For a number of broad classes of stimuli, this is no longer the accepted explanation. For example, many motion illusions can be explained as a consequence of Bayesian inferences being made from noisy data ${ }^{60}$. The present work has extended this to the auditory system, where it is proposed that a simple adaptive mechanism, when driven outside its normal operating regime, may generate uncertainties which makes the optimal interpretation contain illusory

\begin{tabular}{c|cc|c} 
& \multicolumn{2}{|c|}{$\begin{array}{c}\text { Zwicker } \\
\text { Perceived }\end{array}$} & \\
Tinnitus & yes & no & total \\
\hline yes & 10 & 1 & 11 \\
no & 14 & 19 & 33 \\
\hline total & 24 & 20 & 44
\end{tabular}

TABLE I. Number of normal and tinnitus subjects reporting Zwicker tone percept.

percepts. Specifically, the psychophysical and modeling results reported above support the hypothesis that tinnitus and the Zwicker tone may be a consequence of gain adaptation, and that the loss of compressive non-linearity may accentuate and modify these percepts even in the absence of elevated hearing thresholds.

Taken together, the modeling and psychophysics results suggest distinct regimes of operation for normal and tinnitus subjects. It might be reasonably speculated that tinnitus subjects have lost the instantaneous amplification mechanism of outer hair cells in selective bands; that this disrupts the dynamic range compression inherent in the nonlinear amplification mechanism; and that as a result, a slower neuronal gain adaptation mechanism becomes the dominant factor.

\section{A. Evidence related to tinnitus and hearing loss}

In the auditory periphery there are at least two mechanisms that are thought to address the problem of dynamic range mismatch between the auditory nerve fibers, which lies between $20-40 \mathrm{~dB}$, and the dynamic range in the auditory input of about $120 \mathrm{~dB}$. First, outer hair cells are thought to actively amplify faint sounds with large gains, while at high signal intensities the gain is reduced. This nonlinear amplification leads to a compression of dynamic range. Second, inner hair cells are contacted by multiple auditory fibers with different response thresholds and gains. Therefore, as intensity increases an increasing number of fibers are recruited, which effectively increases the available dynamic range of neuronal firing for a group of fibers with a common characteristic frequency.

Peripheral hearing loss is associated with elevated thresholds. This results in a reduced diversity of response thresholds required by the recruitment mechanism. This is thought to be the origin of abnormally fast growth in loudness ${ }^{61}$. In addition, outer hair cell damage, which is often associated with peripheral hearing loss, leads to a loss of active amplification, reducing the compressive effect of the nonlinear cochlear amplifier ${ }^{61}$. Here it is postulated that, when faced with these challenges, downstream mechanisms compensate by taking a more active role in coping with the dynamic range of the input. These mechanisms, when confronted with silence in selected frequency bands, increase internal gains, which then amplify 
neuronal noise to the point that it is perceived as phantom sounds. Tinnitus and the Zwicker tone, in this view, are not associated with increased activity in the periphery, yet are nonetheless ultimately caused by alterations in the peripheral apparatus.

Note that elevated thresholds are a common correlate of tinnitus ${ }^{4}$, and abnormal growth of loudness is observed for frequencies matching the tinnitus percept ${ }^{62}$. In addition, distortion products, which are thought to reflect the operation of the nonlinear cochlear amplifier, are selectively altered for frequency bands that have been matched to the tinnitus percept ${ }^{63-65}$. Finally, release from masking by a secondary masking tone does not occur in tinnitus subjects, indicating once again that the nonlinear effect of this two-tone suppression ascribed to the cochlear amplifier is not operating in tinnitus subjects $^{66,67}$. All this supports the hypothesis that tinnitus is a result of hearing loss and degraded nonlinear compression.

A common strategy to alleviate tinnitus consists of masking the tinnitus percept with acoustic noise in the corresponding frequency band. While this method is effective in eliminating the tinnitus percept for the duration of the noise, it is seldom adopted by patients, as it accomplishes little more than replacing one auditory annoyance by another. Interestingly, a residual inhibition following the masking noise and lasting up to minutes is commonly observed ${ }^{68}$. It has also been reported that hearing aids properly fitted to the frequencies of hearing loss can sometimes alleviate tinnitus ${ }^{3,69,70}$. Some reports indicate that tinnitus can be alleviated on a longer time scale by delivering variable signals in selected frequency bands ${ }^{71}$, in particular after noise-induced hearing loss preempting central adaptation ${ }^{72}$. Perceptual training paradigms aiming at central adaptation mechanisms have also been used to alleviate tinnitus percepts ${ }^{73}$. All this is in good agreement with the hypothesis proposed above, which maintains that the increased gains can be reduced by delivering signal variance to the damaged channel. This moreover suggests that a properly fitted compressive hearing aid may alleviate tinnitus for those subjects where tinnitus is caused by a loss of nonlinear amplification and/or a partial loss of sensitivity.

\section{B. Neural substrate}

The above model of gain adaptation explains the sensitization and release from masking that has been observed psychophysically following notched noise ${ }^{19,20}$. The model makes minimal assumptions about the neural processing required in the gain adaptation mechanism. It assumes that intensity is encoded separately for each frequency band, presumably in neuronal firing rates of a group of neurons, and that the overall loudness of the signal is encoded separately from the intensity of each individual band. Finally, it assumes that signal power can be accumulated over some time frame and that this esti- mate can be used to reduce or inhibit the activity in each band. Most of these assumptions are compatible with the present knowledge of neuronal function.

It has not been necessary above to specify at which level of neural processing the gain adaptation mechanism may be operating. In fact, several stages of adaptation may be possible. For example, the mechanism of gain control could be operating as part of the control of outer hair cell response through medial olivocochlear (MOC) efferent feedback ${ }^{27,74}$. In this context it is interesting to note that it has been reported that the efferent inhibition of outer hair-cell function as evidenced by distortion products is impaired in most tinnitus subjects ${ }^{4}$. A central mechanisms is also consistent with the finding that unilateral cochlear implants generally reduce contralateral tinnitus ${ }^{75}$. Gain could also be adjusted through inhibition and/or excitation of primary afferent nerve fibers through lateral olivocochlear (LOC) efferents ${ }^{76,77}$. A recent review suggests that most current electrophysiology on tinnitus implicates stages upstream of the auditory nerve $^{10}$. Similarly, the only current reports on physiological correlates of the Zwicker tone come from the auditory cortex ${ }^{78,79}$. Most notably, gain adaptation has been demonstrated for inferior colliculus neurons ${ }^{30}$, and is mediated by inhibitory input ${ }^{80}$.

Section II.E points out that after gain normalization, the response in separate frequency bands does not distinguish long-term silence from persistent uniform noise. It is argued that for efficient information transmission, overall loudness is better transmitted as a separate variable which can then be used to disambiguate silence from uniform noise. The questions that begs answering is: what is the neuronal substrate for such a representation? There are many cells in the auditory cortex with high spontaneous activity which respond only transiently with an increase in firing rate to the onset of sound. Few cortical cells respond tonicly to a steady stimulus. A distinct representation between loudness and modulation may therefore not seem unreasonable for the auditory cortex. The situation for the auditory nerve is less straightforward. On the surface it would seem that loudness is encoded in the overall firing rate. Yet in fact, an increase in firing rate does not necessarily reflect an increase in loudness ${ }^{81}$, and firing rate is not a sufficient model to explain level discrimination $^{82}$. Instead, other mechanisms such as synchrony and phase relations across fibers may be required to explain psychophysical performance. It is also conceivable that outer hair cell afferent fibers, which are just now being characterized ${ }^{83}$, serve a role in this regard. ${ }^{84}$ Despite much effort, the details of how overall level is encoded in the auditory nerve remains an open question.

Note that the basic mechanism proposed here works separately for each frequency band, and is therefore uniform across frequencies. In contrast, some reports on the Zwicker tone suggest that the phenomenon is asymmetric, in that subjects tend to match the perception with a tone that is somewhat above the lower edge of the notched band ${ }^{11,13}$. In fact, a high-pass band edge 
may not elicit a Zwicker tone. In addition, a sharp edge is required to elicit the Zwicker tone. Similarly, tinnitus is associated with heightened sensitivity at the edge of high-frequency hearing $\operatorname{loss}^{10}$ and requires that the slope of hearing loss is high ${ }^{1}$. These phenomena have been explained by asymmetric lateral inhibition for the Zwicker tone ${ }^{51,85}$, or a loss of lateral inhibition from a damaged band for tinnitus ${ }^{10}$. Similarly, when asymmetric lateral suppression is included in the model, the percept is more pronounced at a lower frequency edge and non-existent for a sloping edge. However, one might argue that these effects are not a prerequisite to explain the phantom precept. Instead, this manuscript focuses on a simple explanation for a basic mechanism so that robust experimental predictions could be derived.

\section{Logarithmic scale of intensity level}

With the exception of equation (7), the model has been formulated in terms of the powers of the signal, i.e., directly in terms of sound pressure level (SPL). Neuronal signals however typically scale with the logarithm of the SPL, which is therefore typically given in decibel. Within a narrow dynamic range, the conversion from linear SPL to a logarithmic scale occurs at the first stage of signal transduction, i.e., the hair cell ${ }^{86}$. Membrane potentials of hair cells ${ }^{87}$ as well as the resulting firing rates in the auditory nerve ${ }^{88}$ scale with the logarithm of signal power, provided the SPL is within the narrow dynamic range of the specific cell. A logarithmic response over a large dynamic range - as required to explain observed perceptual sensitivity (Weber's law) - is likely to involves a combination of cells with different thresholds and slopes ${ }^{89}$. To avoid making specific assumptions as to how this is accomplished by the auditory system, the gain adaptation model is formulated in the original domain of linear SPL. Only lateral inhibition was formulated in the log-domain, reflecting the fact that it operates at the level of the auditory nerve and further upstream.

In a logarithmic perceptual scale a multiplicative factor $H$ becomes an additive contribution, and the power factor $\beta$ becomes a multiplicative factor. Gain adaptation, as formulated here, corresponds to an adaptation to the mean power of the signal by a horizontal shift in the conventional rate-response function. A loss in compression (increasing slope $\beta$ ) necessitates a correction of the slope, which has not been modeled above, or a fast adaptation of the threshold of the rate-response function. This is the hypothesized mechanism leading to a larger susceptibility to the Zwicker tone for tinnitus subjects.

The present work has analogs to a model for tinnitus developed concurrently ${ }^{90}$, which separately considers damage to inner hair cells (IHC), outer hair cells (OHC), and stereocilia. That model operates on the logarithm of the signal power and assumes that IHC damage is multiplicative on this logarithmic scale, that stereocilia damage is additive thus reducing background noise, and that
OHC damage is additive therefore not affecting background noise. In the model described here, these three forms of loss correspond respectively to a loss in compression (power factor $\beta$ ), a multiplicative loss (the factor $H$ ) with a simultaneous reduction of spontaneous rate (the noise $N$ ), and a pure multiplicative loss (the factor $H$ ). The edge effects discussed above are modeled differently in their work, however the most significant difference between the models is the criterion that leads to the phantom percept: the alternative model ${ }^{90}$ require two levels of adaptation, namely optimal information transmission at the level of the AN followed by homeostatic adaptation to a desired firing rate. The present model, in contrast, only requires optimal information transmission at some stage of processing.

\section{Prediction}

The mechanism proposed here predicts that gain adaptation should vary across frequencies for a given subject depending on the strength of the nonlinear compression at each frequency band. This work therefore predicts a link between sensitization following a notched noise ${ }^{19,20}$ and the various correlates that are commonly associated with the nonlinear effects of the cochlear amplifier, such as distortion products or two-tone suppression-both of which can be measured psychophysically or audiometrically using otoacoustic emissions ${ }^{17,47,91,92}$.

\section{CONCLUSIONS}

Two main conclusions follow from this work. The first is that a rather simple model of optimal auditory adaptation can account for tinnitus as a consequence of a mismatch between the design parameters of the adaptive system and the actual performance of the sensory apparatus. This account does not speak directly as to where in the brain tinnitus arises, but rather as to why. The model makes a novel testable prediction concerning a linkage between Zwicker tone and tinnitus, which was experimentally confirmed. It also predicts a link between the Zwicker tone and cochlear amplification, which will be tested in future experiments. The second conclusion is that, regardless of the motivation for the psychophysical experiments conducted, the experimental results in and of themselves constitute a novel empirical link between tinnitus and Zwicker tone.

${ }^{1}$ O. Konig, R. Schaette, R. Kempter, and M. Gross, "Course of hearing loss and occurrence of tinnitus", Hearing Research (2006).

2 J. C. Cooper, Jr., "Health and nutrition examination survey of 1971-75: Part II. tinnitus, subjective hearing loss, and well-being", J Am Acad Audiol 5, 37-43 (1994).

3 V. Vesterager, "Tinnitus: Investigation and management", British Medical Journal 314, 728-31 (1997). 
${ }^{4}$ G. Hesse, H. Schaaf, and A. Laubert, "Specific findings in distortion product otoacoustic emissions and growth functions with chronic tinnitus", Int Tinnitus J 11, 6-13 (2005).

5 A. Norena, C. Micheyl, S. Chery-Croze, and L. Collet, "Psychoacoustic characterization of the tinnitus spectrum: Implications for the underlying mechanisms of tinnitus", Audiol Neurootol. 7, 358-69 (2002).

${ }^{6}$ R. S. Tyler, ed., Tinnitus Handbock (Thomson, Delmar Learning) (2000).

7 D. M. Baguley, "Mechanisms of tinnitus", British Medical Bulletin 63, 195-212 (2002).

8 A. R. Moller, Hearing: Its Physiology and Pathophysiology (Academic Press) (2000).

9 J. J. Eggermont, "Psychological mechanisms and neural models", in Tyler ${ }^{6}, 85-122$.

10 J. J. Eggermont and L. E. Roberts, "The neuroscience of tinnitus", Trends Neurosci 27, 676-82 (2004).

11 A. Norena, C. Micheyl, and S. Chery-Croze, "An auditory negative after-image as a human model of tinnitus", Hearing Research 149, 24-32 (2000).

12 E. Zwicker, "Negative afterimage" in hearing", J. Acoust. Soc. Am. 36, 2413-5 (1964).

13 E. Zwicker and H. Fastl, Psychoacoustics: Facts and Models (Springer) (1999).

14 H. Fastl, "On the Zwicker-tone of line spectra with a spectral gap", Acustica 67, 177-86 (1989).

15 R. C. Lummis and N. Guttman, "Exploratory studies of Zwicker's "negative afterimage" in hearing", J. Acoust. Soc. Am. 51, 1930-44 (1972).

16 F. Julicher, D. Andor, and T. Duke, "Physical basis of twotone interference in hearing", Proceedings of the National Academy of Sciences of the United States of America 98, 9080-5 (2001).

17 R. V. Shannon and T. Houtgast, "Psychophysical measurements relating suppression and combination tones", J. Acoust. Soc. Am. 68, 825-9 (1980).

18 R. Stoop and A. Kern, "Two-tone suppression and combination tone generation as computations performed by the hopf cochlea", Physical Review Letters 93, 268103 (2001).

19 R. P. Carlyon, "Changes in the masked thresholds of brief tones produced by prior bursts of noise", Hearing Research 41, 223-35 (1989).

${ }^{20}$ L. Wiegrebe, M. Koessl, and S. Schmidt, "Auditory sensitization during the perception of acoustical negative afterimages: Analogies to visual processing?", Naturwissenschaften 82, 387-9 (1995).

21 J. J. Atick and A. N. Redlich, "Quantitative tests of a theory of retinal processing: contrast sensitivity curves", Technical Report NYU-NN-90/2, New York University (1990), also Institute for Advanced Studies IASSNS-HEP$90 / 51$.

22 F. Rieke, D. Warland, R. de Ruyter van Steveninck, and W. Bialek, Spikes: Exploring the Neural Code (MIT Press) (1996), a Bradford Book.

${ }^{23}$ H. B. Barlow, R. Fitzhugh, and S. W. Kuffler, "Change of organization in the receptive fields of the cats retina during dark adaptation", Journal of Physiology 137, 33854 (1957)

${ }^{24}$ I. Ohzawa, G. Sclar, and R. D. Freeman, "Contrast gain control in the cat visual cortex", Nature 289, 266-8 (1982).

25 R. M. Shapley and J. D. Victor, "The contrast gain control of the cat retina", Vision Research 19, 431-4 (1979).

${ }^{26}$ Y. Yu, B. Potetz, and T. S. Lee, "The role of spiking non- linearity in contrast gain control and information transmission", Vision Research 45, 583-92 (2005).

27 J. J. Guinan, Jr., "Physiology of olivocochlear efferents", in The Cochlea, 435-502 (Springer) (1996).

28 R. L. Smith and J. J. Zwislocki, "Short-term adaptation and incremental responses of single auditory-nerve fibers", Biological Cybernetics 17, 169-82 (1975).

${ }^{29}$ L. A. Westerman and R. L. Smith, "Rapid and short-term adaptation in auditory nerve responses", Hearing Research 15, 249-60 (1984).

30 I. Dean, N. S. Harper, and D. McAlpine, "Neural population coding of sound level adapts to stimulus statistics", Nature Neuroscience 8, 1684-9 (2005).

31 N. J. Ingham and D. McAlpine, "Spike-frequency adaptation in the inferior colliculus", Journal of Neurophysiology 91, 632-45 (2004).

32 A. F. Jahn and J. R. Santos-Sacchi, eds., Physiology of the Ear, 2nd edition (Singular) (2001).

33 B. J. C. Moore, An Introduciton to the Psypchology of Hearing, 4th edition (Academic Press) (2003).

${ }^{34}$ I. Nelken, Y. Rotman, and O. Bar Yosef, "Responses of auditory-cortex neurons to structural features of natural sounds", Nature 397, 154-7 (1999).

35 R. V. Shannon, F. G. Zeng, V. Kamath, J. Wygonski, and M. Ekelid, "Speech recognition with primarily temporal cues", Science 270, 303-4 (1995).

36 L. C. Parra, C. D. Spence, and P. Sajda, "Higher-order statistical properties arising from the non-stationarity of natural signals", in Advances in Neural Information Processing Systems 13, 786-92 (2001).

37 J. R. Cavanaugh, W. Bair, and J. A. Movshon, "Selectivity and spatial distribution of signals from the receptive field surround in macaque V1 neurons", Journal of Neurophysiology 88, 2547-56 (2002).

38 J. Malo, I. Epifanio, R. Navarro, and E. P. Simoncelli, "Non-linear image representation for efficient perceptual coding", IEEE Trans. Image Processing 15, 68-80 (2006).

39 R. Valerio and R. Navarro, "Optimal coding through divisive normalization models of V1 neurons", Network 14, 579-93 (2003).

40 R. Buccigrossi and E. P. Simoncelli, "Image compression via joint statistical characterization in the wavelet domain", IEEE Trans. Image Processing 8, 1688-701 (1999).

41 C. E. Shannon, "A mathematical theory of communication", Bell System Technical Journal 27, 379-423, 623656 (1948), URL http://cm.bell-labs.com/cm/ms/what/ shannonday/paper.html.

42 Frequency decomposition generates approximately zeromean normal-distributed data, in which case the optimal transfer function has the shape of a Gaussian CDF centered at zero, and optimal transmission is achieved by adjusting the variance of the input to the slope of the CDF. Alternatively, log-power may be communicated, which is also well approximated by a normal distribution. In this case, overall the signal gain adjusts the mean of the Gaussian so that the mean power matches the center of the CDF. In either case, adjusting the data to have unit variance is the first-order correction to maximize transmitted information.

43 This can be viewed as a trivial Kalman filter; of course, a more sophisticated estimator could be used instead.

${ }^{44}$ M. LeMasurier and P. G. Gillespie, "Hair-cell mechanotransduction and cochlear amplification", Neuron 48, 403-15 (2005). 
45 A. J. Hudspeth, Y. Choe, A. D. Mehta, and P. Martin, "Putting ion channels to work: Mechanoelectrical transduction, adaptation, and amplification by hair cells", PNAS 97, 11765-72 (2000).

46 V. M. Eguiluz, M. Ospeck, Y. Choe, A. J. Hudspeth, and M. O. Magnasco, "Essential nonlinearities in hearing", Physical Review Letters 84, 5232-5 (2000).

47 E. J. Williams and S. P. Bacon, "Compression estimates using behavioral and otoacoustic emission measures", Hearing Research 201, 44-54 (2005).

48 T. Janssen, P. Kummer, and W. Arnold, "Growth behavior of the 2 f1-f2 distortion product otoacoustic emission in tinnitus", J. Acoust. Soc. Am. 103, 3418-30 (1998).

49 R. V. Harrison, "Rate-versus-intensity functions and related AP responses in normal and pathological guinea pig and human cochleas.", J. Acoust. Soc. Am. 70, 1036-44 (1981).

50 R. S. Tyler and D. Conrad-Armes, "Masking of tinnitus compared to masking of pure tones", J Speech Hear Res 27, 106-11 (1984).

51 J. M. Franosch, R. Kempter, H. Fastl, and J. L. van Hemmen, "Zwicker tone illusion and noise reduction in the auditory system", Physical Review Letters 90, 178103 (2003).

${ }^{52}$ G. M. Gerken, "Central tinnitus and lateral inhibition: An auditory brainstem model", Hearing Research 97, 75-83 (1996).

53 W. S. Rhode and S. Greenberg, "Lateral suppression and inhibition in the cochlear nucleus of the cat", Journal of Neurophysiology 71, 493-514 (1994).

${ }^{54}$ U. W. Biebel and G. Langner, "Evidence for interactions across frequency channels in the inferior colliculus of awake chinchilla", Hear Res 169, 151-68 (2002).

${ }^{55}$ M. L. Sutter, C. E. Schreiner, M. McLean, K. N. O'Connor, and W. C. Loftus, "Organization of inhibitory frequency receptive fields in cat primary auditory cortex", J Neurophysiol 82, 2358-71 (1999).

56 Example signals are available at http://newton.bme. columbia. edu/ lparra/tinnitus/. For these listening examples high frequencies are de-emphasized in the reconstructed signal by scaling the power at frequency $\omega$ by $1 / \omega$.

57 R. D. Patterson and B. C. J. Moore, "Auditory filters and excitation patterns as representations of frequency resolution", in Frequency Selectivity in Hearing, edited by B. C. J. Moore, chapter 3, 123-77 (Academic Press) (1986).

58 M. Slaney, "An efficient implementation of the PattersonHoldsworth cochlear filter bank", Technical Report 45, Apple, Cupertino, CA (1993), URL http://www.slaney .org/ malcolm/pubs.html, Auditory Toolbox version 2.0.

59 This subjective criterion was used because currently there is no objective test available for tinnitus.

${ }^{60}$ Y. Weiss, E. P. Simoncelli, and E. H. Adelson, "Motion illusions as optimal percepts", Nature Neuroscience 5, 598604 (2002).

61 M. G. Heinz and E. D. Young, "Response growth with sound level in auditory-nerve fibers after noise-induced hearing loss", Journal of Neurophysiology 91, 784-95 (2004).

62 M. J. Penner, "Magnitude estimation and the "paradoxical" loudness of tinnitus", J Speech Hear Res 29, 407-12 (1986)

63 H. Gouveris, J. Maurer, and W. Mann, "DPOAE-grams in patients with acute tonal tinnitus", Otolaryngol Head
Neck Surg 132, 550-3 (2005).

64 E. T. Onishi, Y. Fukuda, and F. A. Suzuki, "Distortion product otoacoustic emissions in tinnitus patients", Int Tinnitus J 10, 13-6 (2004).

65 Y. Shiomi, J. Tsuji, Y. Naito, N. Fujiki, and N. Yamamoto, "Characteristics of DPOAE audiogram in tinnitus patients", Hearing Research 108, 83-8 (1997).

66 C. R. Mitchell and T. A. Creedon, "Psychophysical tuning curves in subjects with tinnitus suggest outer hair cell lesions", Otolaryngol Head Neck Surg 113, 223-33 (1995).

67 M. J. Penner, "Two-tone forward masking patterns and tinnitus", J Speech Hear Res 23, 779-86 (1980).

68 A. M. Terry, D. M. Jones, B. R. Davis, and R. Slater, "Parametric studies of tinnitus masking and residual inhibition", Br J Audiol 17, 245-56 (1983).

69 J. Vernon, "Attempts to relieve tinnitus", J Am Audiol Soc 2, 124-31 (1977).

70 J. Vernon and M. B. Meikle, "Tinnitus masking", in Tyler $^{6}, 313-55$.

71 B. A. Goldstein, A. Shulman, M. L. Lenhardt, D. G. Richards, A. G. Madsen, and R. Guinta, "Long-term inhibition of tinnitus by UltraQuiet therapy: Preliminary report", Int Tinnitus J 7, 122-7 (2001).

72 A. J. Norena and J. J. Eggermont, "Enriched acoustic environment after noise trauma reduces hearing loss and prevents cortical map reorganization", Journal of Neuroscience 25, 699-705 (2005).

73 C. Formby, L. P. Sherlock, and S. L. Gold, "Adaptive plasticity of loudness induced by chronic attenuation and enhancement of the acoustic background", J. Acoust. Soc. Am. 114, 55-8 (2003).

74 J. J. Guinan, Jr. and M. L. Gifford, "Effects of electrical stimulation of efferent olivocochlear neurons on cat auditory-nerve fibers I: Rate-level functions", Hearing Research 33, 97-113 (1988).

75 N. Quaranta, S. Wagstaff, and D. M. Baguley, "Tinnitus and cochlear implantation", Int J Audiol. 43, 245-51 (2004).

76 J. A. Groff and M. C. Liberman, "Modulation of cochlear afferent response by the lateral olivocochlear system: Activation via electrical stimulation of the inferior colliculus", Journal of Neurophysiology 90, 3178-200 (2003).

77 W. H. Mulders and D. Robertson, "Diverse responses of single auditory afferent fibres to electrical stimulation of the inferior colliculus in guinea-pig", Experimental Brain Research 160, 235-44 (2005).

78 E. S. Hoke, B. Ross, and M. Hoke, "Auditory afterimage: Tonotopic representation in the auditory cortex", Neuroreport 9, 3065-8 (1998).

79 A. J. Norena and J. J. Eggermont, "Neural correlates of an auditory afterimage in primary auditory cortex", J Assoc Res Otolaryngol 4, 312-28 (2003).

80 N. J. Ingham and D. McAlpine, "GABAergic inhibition controls neural gain in inferior colliculus neurons sensitive to interaural time differences", Journal of Neuroscience 25, 6187-98 (2005).

81 E. M. Relkin and J. R. Doucet, "Is loudness simply proportional to the auditory nerve spike count?", J. Acoust. Soc. Am. 101, 2735-40 (1997).

82 H. S. Colburn, L. H. Carney, and M. G. Heinz, "Quantifying the information in auditory-nerve responses for level discrimination", J Assoc Res Otolaryngol 4, 294-311 (2003).

83 M. A. Reid, J. Flores-Otero, and R. L. Davis, "Firing pat- 
terns of type II spiral ganglion neurons in vitro", Journal of Neuroscience 24, 733-42 (2004).

84 Their sparseness, convergent connectivity, slower responses, reduced accommodation, and association with the cochlear amplifiers make the outer hair cell afferent fibers ideal candidates to encode loudness.

85 A. Kral and V. Majernik, "On lateral inhibition in the auditory system", Gen Physiol Biophys 15, 109-27 (1996).

86 C. J. Sumner, E. A. Lopez-Poveda, L. P. O'Mard, and R. Meddis, "A revised model of the inner-hair cell and auditory-nerve complex", J. Acoust. Soc. Am. 111, 217888 (2002).

87 P. Dallos, "Response characteristics of mammalian cochlear hair cells", Journal of Neuroscience 5, 1591-608 (1985).

88 B. Sachs, Murray and P. J. Abbas, "Rate versus level function for autitory-nerve fibers in cat: Tone-burst stimuli",
J. Acoust. Soc. Am. 56, 1835-47 (1974).

89 M. Winter, Ian and R. Palmer, Alan, "Intensity coding in low-frequency auditory-nerve fibers of the guinea pig", J. Acoust. Soc. Am. 90, 1958-67 (1991).

90 R. Schaette and R. Kempter, "Development of tinnitusrelated neuronal hyperactivity through homeostatic plasticity after hearing loss: A computational model", European Journal of Neuroscience 23, 3124-38 (2006).

91 A. J. Oxenham and C. J. Plack, "A behavioral measure of basilar-membrane nonlinearity in listeners with normal and impaired hearing", J. Acoust. Soc. Am. 101, 3666-75 (1997).

92 C. J. Plack and A. J. Oxenham, "Basilar-membrane nonlinearity estimated by pulsation threshold", J. Acoust. Soc. Am. 107, 501-7 (2000). 